\title{
Separate Areas for Mirror Responses and Agency within the Parietal Operculum
}

\author{
Zarinah Agnew ${ }^{1}$ and Richard J. S. Wise ${ }^{1,2}$ \\ ${ }^{1}$ Cognitive Neuroimaging Group, Medical Research Council Clinical Sciences Centre, and ${ }^{2}$ Division of Neuroscience and Mental Health, Imperial College \\ London, Hammersmith Hospital Campus, London W12 0NN, United Kingdom
}

There is common neural activity in parietal and premotor cortex when executing and observing goal-directed movements: the "mirror" response. In addition, active and passive limb movements cause overlapping activity in premotor and somatosensory cortex. This association of motor and sensory activity cannot ascribe agency, the ability to discriminate between self- and non-self-generated events. This requires that some signals accompanying self-initiated limb movement dissociate from those evoked by observing the action of another or by movement imposed on oneself by external force. We demonstrated associated activity within the medial parietal operculum in response to feedforward visual or somatosensory information accompanying observed and imposed finger movements. In contrast, the response to motor and somatosensory information during self-initiated finger and observed movements resulted in activity localized to the lateral parietal operculum. This ascribes separate functions to medial and lateral second-order somatosensory cortex, anatomically dissociating the agent and the mirror response, demonstrating how executed and observed events are distinguished despite common activity in widespread sensorimotor cortices.

Key words: fMRI; action; agency; parietal operculum; mirror neuron; motor

\section{Introduction}

Executed, observed, and imagined actions and sensations are encoded in a similar manner (Fadiga et al., 1995; Grèzes et al., 2003; Keysers and Perrett, 2004; Decety and Grèzes, 2006). This is attributed to the function of mirror neurons (di Pellegrino et al., 1992). Much activity associated with self-initiated motor acts represents processing of polysensory (somatosensory and visual) feedback afferent information. Thus, actively and passively initiated movements overlap in terms of their functional anatomy (Weiller et al., 1996; Guzzetta et al., 2007). The present study has investigated the neural systems that distinguish between these movements. This is central to "agency"; the ability to distinguish between movements of internal and external origin.

A forward model of motor control forms the basis for a theory of agency attribution (Wolpert et al., 1995; Frith et al., 2000). It is suggested that an efferent motor command is accompanied by a replica command, the efference copy (von Holst, 1954). The existence of efference copies is well established in animal research (Crapse and Sommer, 2008). The feedforward efference copy acts as a reference against which feedback afferent information can be compared. Although this system monitors and controls actions, it may also contribute to the sense of ownership of an action, or agency (Wolpert and Flanagan, 2001): the absence of an efference

\footnotetext{
Received June 18, 2008; revised Sept. 24, 2008; accepted Sept. 29, 2008

We thank Dr. Kishore Bhakoo and Prof. Basant Puri for their help with this study.

Correspondence should be addressed to Zarinah Agnew, Cognitive Neuroimaging Group, Medical Research Council Clinical Sciences Centre, Imperial College London, Hammersmith Hospital Campus, London W12 0NN, UK. E-mail: z.agnew@imperial.ac.uk.

DOI:10.1523/JNEUROSCI.2836-08.2008

Copyright $\odot 2008$ Society for Neuroscience $\quad$ 0270-6474/08/2812268-06\$15.00/0
}

copy signaling that an event is externally generated (Wolpert et al., 1995). In addition, when the sensory consequences of a selfgenerated event are predictable, responses to reafferent feedback are suppressed. This sensory gating (Blakemore et al., 1998; Roy and Cullen, 2004) is thought to depend on an interaction between cerebral and cerebellar cortical areas (Blakemore et al., 1999).

In summary, although polysensory experiences during active, passive, and observed action are, in large part, commonly encoded in the brain, differential responses to sensory experiences originating from different agents may be responsible for distinguishing the agent. For example, motor facilitation during action observation is only present during the observation of actions by others and not during the observation of one's own actions (Schütz-Bosbach et al., 2006). We investigated execution-evoked activity without visual feedback, to avoid this effect, and compared it with activity generated during observation of action. We were particularly interested in the role of the parietal operculum during self-initiated and observed movements, following a recent magnetoencephalography study (Avikainen et al., 2002). The authors discussed their results in relation to mirror neuron theory but also indicated that suppression of activity in secondary somatosensory cortex (SII) may contribute to the sense of agency.

The present functional imaging study was designed to investigate dissociation of activity within parietal opercular cortex that would differentiate mirror and agency responses. We predicted that a system distinguishing an internal agent would be active during self-generated movement but not during observed or passive movements and vice versa (Blakemore et al., 1998; Wolpert and Flanagan, 2001). Using self-initiated, observed and externally imposed finger movements, we demonstrated the response 
within lateral SII displayed a "mirror" response, whereas medial SII discriminated between the internal and external agent.

\section{Materials and Methods}

Subjects. Twenty healthy right-handed subjects (mean age, 29 years; range, 24-50 years; eight female) participated in this study. All gave informed consent according to the guidelines approved by Hammersmith Hospital Ethics Committee who provided local ethics approval for this study.

Experimental paradigm. In the scanner, subjects were able to see a restricted area around their lower body using a mirror mounted on the head coil. By raising their knees slightly, a board could be placed on their lap, ensuring that actions were performed within their visual field. Subjects' hands were placed by their sides on a foam platform outside of their visual field. This allowed them to perform hand movements without seeing their hands or the experimenter. Subjects were manually cued to performed a self-paced finger tap ("ActiveV-"), observe the experimenter doing the same ("ObserveV+"), or had a finger tap imposed on them ("PassiveV-"). The passive tap was performed by the experimenter using a preplaced finger support to move the subject's finger. Care was taken to ensure that the subject was unable to see any movement during this condition. The support used minimal contact with the subject's finger and was placed approximately half an hour before experimentation to allow for habituation. Alternatively subjects had to view the experimenter's static hand when placed in their visual field ("Stat$\mathrm{icV}+$ ") or a low level baseline of a stationary background ("BaselineV+"). To emphasize, only the ObserveV+, StaticV+, and BaselineV + had visual input. A specular hand was used because this has proved to elicit a stronger mirror response (Koski et al., 2003). We compared brain blood-oxygen level-dependent (BOLD) responses to active action, passive action, and action observation simple finger tapping in a block design paradigm. Each scan consisted of the five conditions repeated four times. Each condition lasted for $21 \mathrm{~s}$ and was separated by a 6 s instruction block. The blocks were organized in pseudorandom order. The entire task lasted $<9$ min.

Scanning. A 3T Philips system was used to acquire $226 \mathrm{~T}_{2}{ }^{*}$-weighted echo-planer images data $\left(2.2 \times 2.2 \times 2.75 \mathrm{~mm}^{3}\right.$; repetition time, 3000 ms; echo time, $30 \mathrm{~ms}$; flip angle, $90^{\circ}$ ) using BOLD contrast. An eightchannel array coil and SENSE (sensitivity encoding) factor 2 were used as well as second-order shims. Two hundred twenty-six functional volumes were acquired from each session, the first five of which were discarded to remove the effect of $\mathrm{T}_{1}$ equilibration. $\mathrm{T}_{2}$ anatomical volume images were also acquired for each subject.

Preprocessing and analyses. Four blocks of each condition were performed resulting in $84 \mathrm{~s}$ of continuous whole-brain acquisition for each condition. Functional data were analyzed using SPM5 (Wellcome Department of Imaging Neuroscience, London, UK) running on Matlab 7.2 (MathWorks). All functional images were realigned to the first volume by six-parameter rigid body spatial transformation. Functional and structural $\left(\mathrm{T}_{2}\right.$-weighted) images were then normalized into standard space using the Montreal Neurological Institute (MNI) template. Functional images were then coregistered to the $\mathrm{T}_{2}$ structural image and smoothed using a Gaussian kernel of full-width half-medium at $8 \mathrm{~mm}$. The data were high-pass filtered at $128 \mathrm{~Hz}$. First-level analysis was performed using motion parameters as regressors of no interest at the singlesubject level. A random-effects model was used in which the data were corrected for multiple comparisons using the false discovery rate (FDR) at a threshold of $p<0.05$ to limit false positives (Curran-Everett, 2000; Genovese et al., 2002). Voxelwise thresholding was performed at 20 voxels to limit potential type II errors.

Individual contrasts were performed to investigate the BOLD response to each condition minus baseline. Given the current debate over conjunction analyses (Friston et al., 2005; Nichols et al., 2005), identification of voxels common to execution and observation conditions was performed using implicit masking at the group level. Significant BOLD effects from this implicit masking analysis were superimposed on a $\mathrm{T}_{2}$ weighted image from one of our volunteers normalized to standard space using the MNI 152 template or rendered on a normalized template. Local foci of maximal activation were then identified using cytoarchitechtonic and probabilistic atlases available within SPM5 (Eickhoff et al., 2005). Region of interest (ROI) analysis was performed to investigate mean effect sizes in specific regions across all experimental conditions against baseline. ROIs of $4 \mathrm{~mm}$ were selected from peak activations in the random-effect analysis and created using the MarsBar toolbox that is available for use within SPM5 (Brett et al., 2002)

\section{Results}

\section{Activity associated with active and passive conditions}

The distributed activity associated with self-initiated finger tapping is displayed in Figure 1. Within left primary sensorimotor cortex, there were separate peaks in primary motor [Brodmann's area 4 (BA 4)] and somatosensory (BA 2) cortices (Geyer et al., 1996). There was accompanying activity in both parietal opercula. On the left, this included both the medial and lateral extent (Eickhoff et al., 2006), whereas on the right, activity was only observed in the lateral operculum (which was apparent even when the threshold was lowered to FDR of $p<0.2$ ). There was additional bilateral activity in more posterior parts of the inferior parietal lobes.

As expected, there was extensive activity in prefrontal, premotor, and subcortical areas. This included the left and right ventrolateral premotor cortex, at the junction of BA 6 and BA 44, the left and right frontal operculum, midline premotor cortex within the supplementary and presupplementary motor areas, and motor cingulate cortex. In subcortical structures, activity surviving the statistical threshold was observed in both caudate nuclei, the left and right pallidum, the right putamen, and bilateral cerebellum (lobules IV-V).

Passive movement of the right index finger revealed activity in left sensory cortex (BA 1 and BA 2), with some activity in the homotopic region on the right (Geyer et al., 1999, 2000). This was accompanied by activity in the left and right parietal operculum, within second-order somatosensory cortex (Eickhoff et al., 2006).

A direct comparison of the ActiveV - and PassiveV - conditions was also performed (Fig. 2). When active was compared with passive, the main outcome was strong activity within the midline cerebellum. There was evidence of greater activity in left primary motor cortex, but, for the many other premotor, prefrontal and parietal regions activity was not significantly greater. When PassiveV - was compared with ActiveV-, the differences were confined to both parietal lobes. There was greater activity during PassiveV - in both the left and right parietal operculum and within left BA 1 and BA 2.

\section{Activity associated with observed conditions relative to static} The contrast of ObservedV + with StaticV + controlled for activity in all visual processing areas, except for left and right visual area 5 (V5), cortex specialized for processing visual motion (Eickhoff et al., 2005). In addition, there was prominent activity in left and right parietal operculum (Fig. 1).

\section{Active, passive, and observed finger movements share common neural responses}

We observed common activity for ActiveV-, PassiveV-, and ObserveV + in bilateral parietal operculum (SII), and cerebellum. Mirror responses, as defined as significant BOLD responses during action execution and action observation, were seen in the lateral part of both parietal opercula. Conversely, the profile of activity in the medial parietal opercula reflected that of a network that discriminates between internal and external agents; that is, there was activity associated with observation and passive finger 


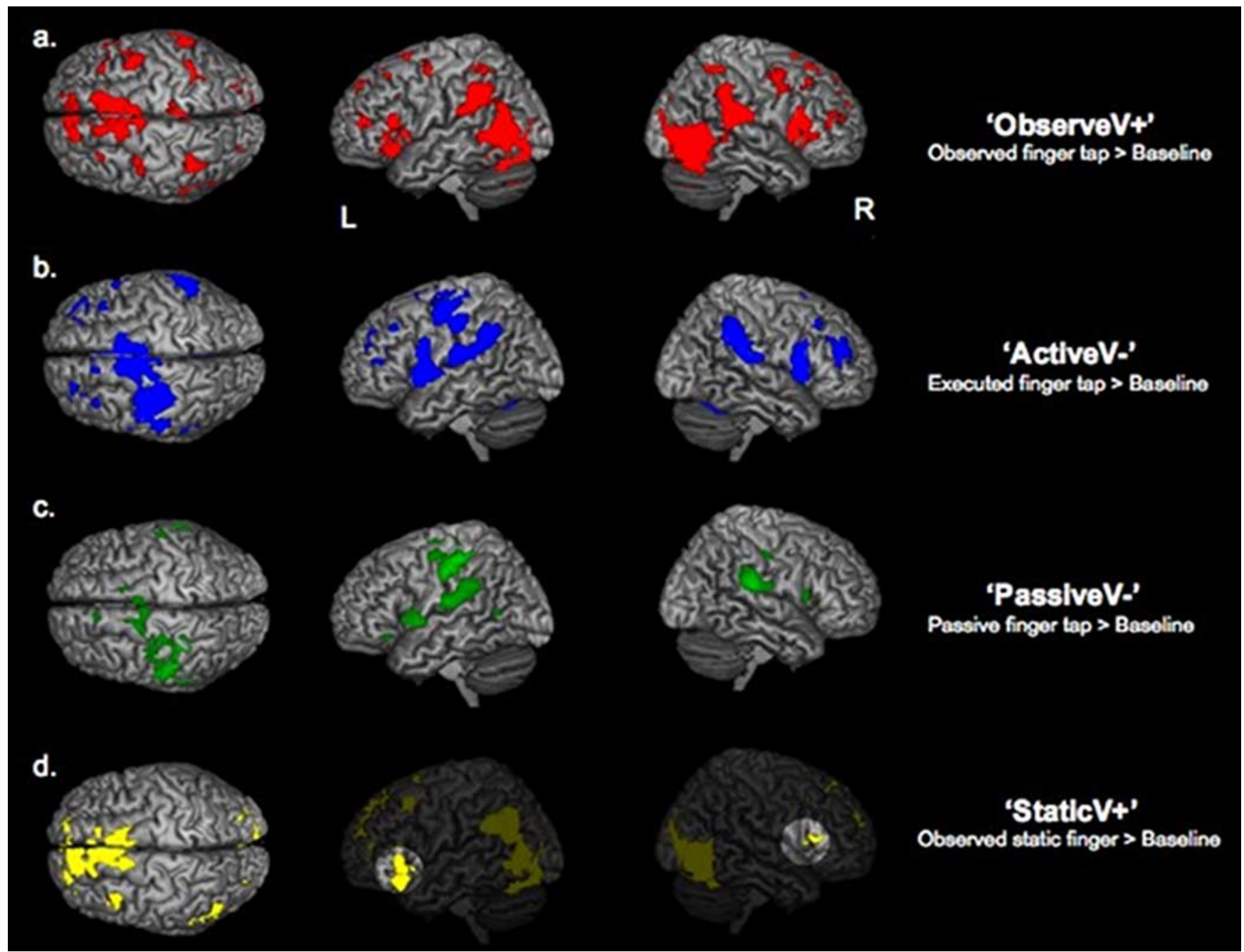

Figure 1. Neural activations associated with Observe, Execute, and Passive conditions are widely overlapping. During the observation of a finger tap versus a Rest condition (a), activity was observed in bilateral parietal operculum, anterior parietal, inferior frontal gyri, and visual cortices, including visual cortical area V5/middle temporal visual motion areas (all contrasts at FDR of 0.05 , extent 20). Active finger tapping $(\boldsymbol{b})$ was accompanied by additional activations in ipsilateral cerebellum, contralateral primary motor cortex, and supplementary motor area. Passively imposed finger movements also resulted in increased activity in similar areas, including contralateral somatosensory cortices, supplementary motor area, parietal operculum, and inferior frontal gyri (c). The Static condition involved observing a static hand against observing a static background that was accompanied by increased activity in visual cortices and bilateral inferior frontal gyri (highlighted). L, Left; R, right.

movements but not self-generated finger movements. This dissociation between mirror response and agency networks is shown in Figure 3.

\section{Discussion}

\section{The parietal opercula}

This study investigated how executed, observed, and passive actions are distinguished, despite the associated sensory experiences being, in part, commonly encoded. Our data showed that adjacent regions within the right parietal operculum differentially encoded a mirror response and agency. A bilateral mirror response, defined as voxels commonly activated in executing and observing a finger tap, contrasted with observing a static hand, was observed in a caudolateral part of the parietal opercula, supporting previous findings (Keysers et al., 2004). Based on a human cytoarchitechtonic map and atlas (Eickhoff et al., 2005, 2006), this mirror response occurred in so-called operculum 1 (OP1) of second-order somatosensory cortex. It is proposed that this is the homolog of monkey SII (Eickhoff et al., 2006). A system that distinguishes between internal and external agents, demon- strated as a difference in activity between a finger tap that was either observed or externally imposed and a self-generated finger tap, was observed in a distinct rostromedial part of the right parietal operculum. This may lie in a region known in the monkey as parietal ventral area (PV), part of second-order somatosensory cortex that has a somatotopy independent of, but complementary to, that of SII and labeled OP4 by Eickhoff et al. (2006).

The functional reasons for two adjacent somatosensory maps in monkey parietal operculum is unknown, but the present study has shown a clear functional distinction in the human. Mapping of human parietal operculum has revealed that the hand representation in SII and PV lie adjacent to each other, with PV lying rostral to SII (Disbrow et al., 2000), as do our dissociated peak activations. It is proposed that these adjacent areas have different patterns of connections, but they communicate with each via direct projections (Disbrow et al., 2002). The observed dissociation confirms that different areas within secondary somatosensory cortex distinguish the agent of internal and external events, with functional anatomical dissociations in the responses to ac- 


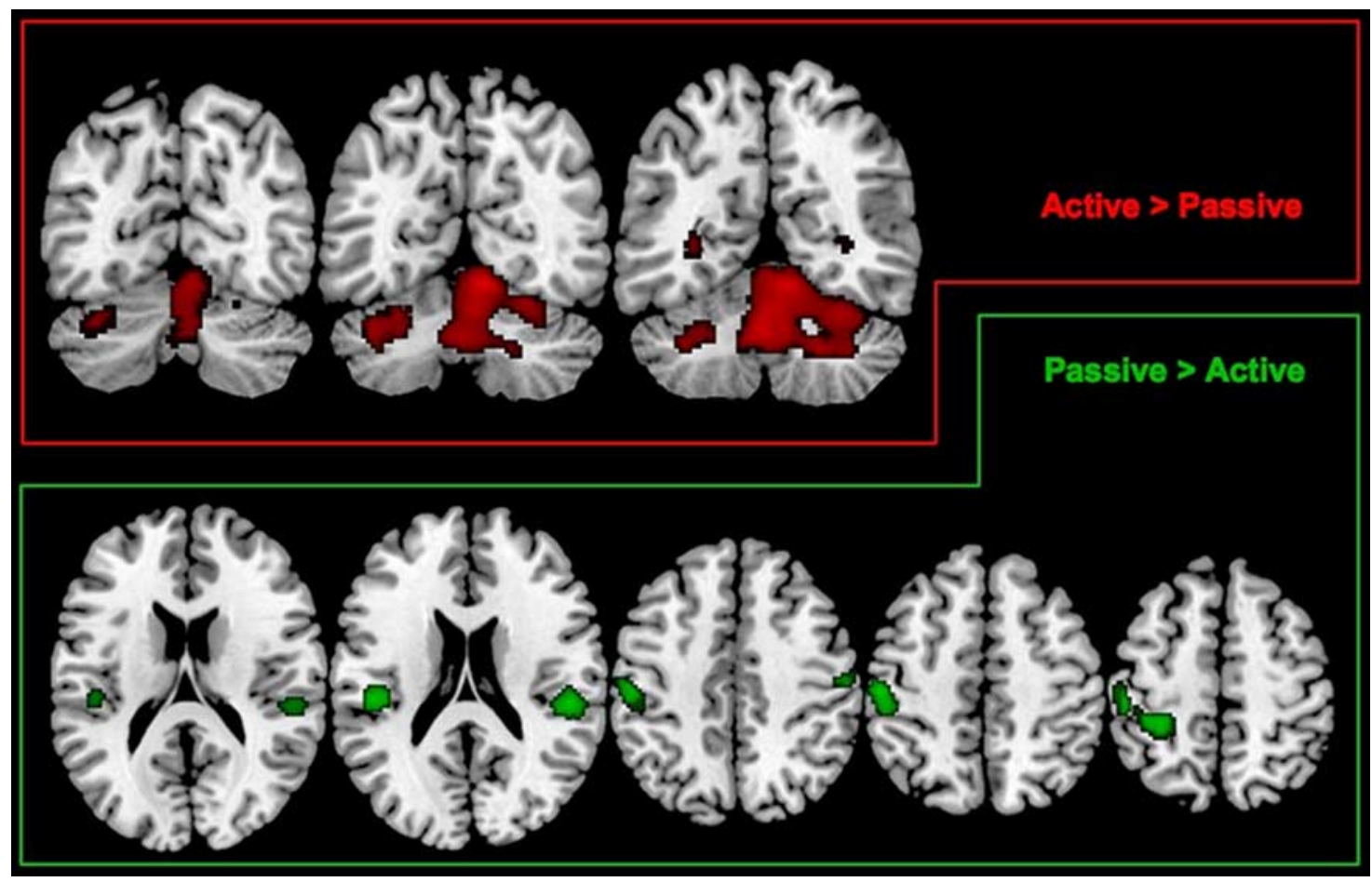

Figure 2. Active movements are associated with right cerebellar activity compared with passive movements. Contrasting BOLD responses during blocks of active finger tapping with blocks of passive finger tapping reveals a right lateralized cerebellar activation that is specific to self-generated action (red; $4,-50,-20)$. The opposite contrast that identifies patterns of BOLD activity that are more correlated with passive rather than active finger tapping are seen in bilateral parietal operculum and left anterior parietal lobes (blue) corresponding to area 2 ( $n=20 ;$ FDR of $0.01 ; k=$ $20 ; 48,-30,26 ; 32,-36,62 ;-52,-22,50 ;-46,-28,24)$. The consequential afferent input in both conditions is the same, and thus the presence of somatosensory processing in Passive with respect to Execute demonstrates that, during self-generated action, sensory processing is suppressed or "gated."

tive, passive, and observed actions (Avikainen et al., 2002; Schütz-Bosbach et al., 2006).

The dissociation reflects the interaction between feedforward and feedback information. Feedforward processes are based on internal models of action and efferent discharges. An ensuing action generates afferent feedback that is required for action monitoring and modification (Kawato, 1999), until its execution becomes optimal (Desmurget and Grafton, 2000). In contrast, passive and observed actions are not associated with a motor command and internal efference copy and involve only reafferent processing. Feedback processing involves area 2 of the postcentral gyrus (Seidler et al., 2004), an area anatomically connected to the parietal operculum. Our results indicated that medial and lateral aspects of the parietal operculum differentially process the contributions from feedforward and feedback processes. OP1 is known to share connections with primary sensory areas 1,2 , and 3 and, farther afield, with premotor cortex (area 6 and 44) and insular cortex in the macaque (Cipolloni and Pandya, 1999). These connections between sensory and motor areas are necessary for the Hebbian development of mirror neuron responses (Keysers and Perrett, 2004; Catmur et al., 2007).

Although the dissociation that we report was only significant in the right hemisphere, the mirror response was bilateral. Recording studies imply that neurons in this region have bilateral receptive fields in response to visual and tactile stimulation (Whitsel et al., 1969; Robinson and Burton 1980). This lateralized effect could have arisen from the arrangement of the experimental contrasts; for voxels to achieve significance in the agency contrast, they had to be more active during observed and passive movements compared with the active execution condition. The self-generated right finger tap may have resulted in a relative asymmetry of activity within the parietal opercula, and this asymmetry masked the dissociation of response within the left parietal operculum. Nevertheless, despite this caveat about the apparent asymmetry in our study, it does accord with evidence suggesting that a right frontoparietal network is responsible for selfreferential encoding (Uddin et al., 2007).

\section{Sensory gating during self-generated action}

Passive finger tapping was associated with increased activity in the right parietal operculum and bilateral anterior parietal cortex compared with self-generated finger tapping. The reduced response to the active condition has been referred to as sensory gating (Blakemore et al., 2001). Although a magnetoencephalography study came to the opposite conclusion, namely that activity in SII is suppressed during observed movement, that study differed markedly in design and so is difficult to compare directly with the present study (Avikainen et al., 2002).

Blakemore et al. (1998) argue that sensory gating occurs when the predicted sensory consequence of a self-generated event (corollary discharge) matches the actual sensory consequence (reafference). Somatosensory activity is suppressed and awareness of the sensory consequence is reduced. Our data confirmed this finding in a simple motor task and showed the same effect in relation to observation of the same motor task. We propose that increased activity in parietal operculum during externally produced events $(\mathrm{ObserveV}+$ and $\mathrm{PassiveV}-$ ) relative to internally generated events (ActiveV-) formed a component of the signal that distinguished between events originating internally and externally.

There are a number of studies that have identified other networks associated with an explicit "sense of agency"; these include angular gyrus (Farrer et al., 2008), insula (Farrer et al., 2003), and 


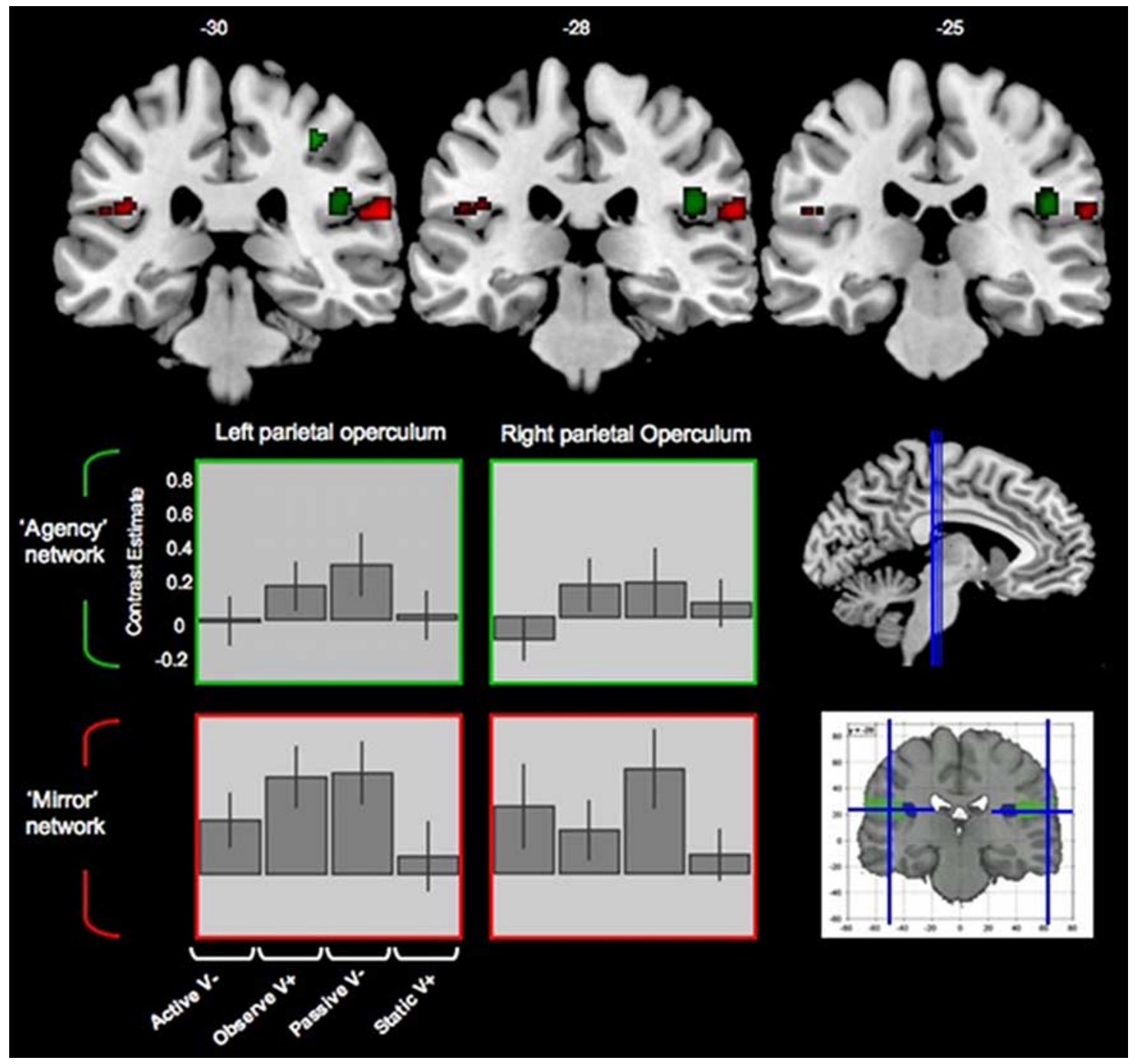

Figure 3. Distinct regions within parietal operculum encode mirror responses and agency. Inclusive masking was used to identify voxels common to both Observe and Active conditions with Static as a baseline condition. Clusters of 20 voxels or more that displayed this profile were defined as exhibiting a mirror response. Such mirror responses, seen in red, were observed in bilateral parietal operculum (inclusive masking at 0.00095 ; FDR of 0.05 , spatial extent 20; at $62,-30,20$ and at $-50,-28,22$ ). To highlight the networks that discriminates between internal and external agents, seen in blue, voxels active during externally originating events were contrasted with self-generated actions (Observe - Execute inclusively masked by Passive - Execute, inclusively masked at 0.00095 ; FDR of 0.05 , spatial extent 20; peak activation at 46, -22 , 22, which was used for region of interest analysis along with a symmetrical regions at $-46,-22,22)$. Graphs display mean contrast estimates at these peak coordinates for all conditions against Baseline condition. Regions of interest were taken using a sphere of $4 \mathrm{~mm}$ radius at each functional peak). Note that, in the lateral extent of parietal operculum, the mirror response, Execute is down with respect to Baseline and Static. Inset is taken from the probabilistic atlas used to localize these regions; in this image, green refers are OP1 (Eickhoff et al., 2006).

posterior parietal cortex (MacDonald and Paus, 2003). The subjective sensation of owning an action is a cognitively different, top-down process to that identified here. However, the evidence indicates that agency may be both top-down and bottom-up (Jeannerod, 2008). In the present study, the passive movement condition was an implicit rather than explicit task and did not involve any manipulation of a sense of ownership in the manner that other studies have used. To emphasize, our study has specifically investigated bottom-up processes involved in agency.

\section{Cerebellar efference copy}

Active finger tapping was associated with greater activity in the right cerebellum and basal ganglia compared with passive finger tapping. This confirms previous findings investigating selfproduced tactile stimulation (Blakemore et al., 1998) and movements (Menon et al., 1998) versus externally produced sensations. This further supports theories of cerebellar forward control, which stipulate that the cerebellum is the generator of the efference copy, along with the motor command. However, although Blakemore et al. (1999) infer that sensory gating of self-produced sensations in sensory cortices is driven by a cerebellar system, there are confounds. The cerebellum is known to play an important role in perception (Fierro et al., 2007; Lee et al., 2007; Bueti et al., 2008) and the timing of temporal intervals in repetitively performed actions (Ivry et al., 2002). The present study unavoidably incorporated the same confounds, and so the observed cerebellar activity can be interpreted in a number of ways, one being that it reflects the formation of a motor efference copy.

\section{Concluding remarks}

This is the first functional imaging study that has investigated dissociations and associations of activity during executed, observed, and passive hand movements. We demonstrated, within the parietal opercula, that caudolateral activity encodes the mir- 
ror response and that rostromedial activity discriminates between internal and external agents.

\section{References}

Avikainen S, Forss N, Hari R (2002) Modulated activation of the human SI and SII cortices during observation of hand actions. Neuroimage 15:640-646.

Blakemore SJ, Wolpert DM, Frith CD (1998) Central cancellation of selfproduced tickle sensation. Nat Neurosci 1:635-640.

Blakemore SJ, Wolpert DM, Frith CD (1999) The cerebellum contributes to somatosensory cortical activity during self-produced tactile stimulation. Neuroimage 10:448-459.

Blakemore SJ, Frith CD, Wolpert DM (2001) The cerebellum is involved in predicting the sensory consequences of action. Neuroreport 12:1879-1884.

Brett M, Anton JL, Valabregue R, Poline JB (2002) Region of interest analysis using an SPM toolbox. Presented at the 8th International Conference on Functional Mapping of the Human Brain, Sendai, Japan, June.

Bueti D, Walsh V, Frith C, Rees G (2008) Different brain circuits underlie motor and perceptual representations of temporal intervals. J Cogn Neurosci 20:204-214.

Catmur C, Walsh V, Heyes C (2007) Sensorimotor learning configures the human mirror system. Curr Biol 17:1527-1531.

Cipolloni PB, Pandya DN (1999) Cortical connections of the frontoparietal opercular areas in the rhesus monkey. J Comp Neurol 403:431-458.

Crapse TB, Sommer MA (2008) Corollary discharge across the animal kingdom. Nat Rev Neurosci 9:587-600.

Curran-Everett D (2000) Multiple comparisons: philosophies and illustrations. Am J Physiol Regul Integr Comp Physiol 279:R1-R8.

Decety J, Grèzes J (2006) The power of simulation: imagining one's own and other's behavior. Brain Res 1079:4-14.

Desmurget M, Grafton S (2000) Forward modeling allows feedback control for fast reaching movements. Trends Cogn Sci 4:423-431.

di Pellegrino G, Fadiga L, Fogassi L, Gallese V, Rizzolatti G (1992) Understanding motor events: a neurophysiological study. Exp Brain Res 91:176-180.

Disbrow E, Roberts T, Krubitzer L (2000) Somatotopic organization of cortical fields in the lateral sulcus of Homo sapiens: evidence for SII and PV. J Comp Neurol 418:1-21.

Disbrow E, Litinas E, Recanzone GH, Slutsky D, Krubitzer L (2002) Thalamocortical connections of the parietal ventral area $(\mathrm{PV})$ and the second somatosensory are (S2) in macaque monkeys. Thalamus Relat Syst $1: 289-302$.

Eickhoff SB, Stephan KE, Mohlberg H, Grefkes C, Fink GR, Amunts K, Zilles K (2005) A new SPM toolbox for combining probabilistic cytoarchitectonic maps and functional imaging data. Neuroimage 25:1325-1335.

Eickhoff SB, Amunts K, Mohlberg H, Zilles K (2006) The human parietal operculum. II. Stereotaxic maps and correlation with functional imaging results. Cereb Cortex 16:268-279.

Fadiga L, Fogassi L, Pavesi G, Rizzolatti G (1995) Motor facilitation during action observation: a magnetic stimulation study. J Neurophysiol 73:2608-2611.

Farrer C, Franck N, Georgieff N, Frith CD, Decety J, Jeannerod M (2003) Modulating the experience of agency: a positron emission tomography study. Neuroimage 18:324-333.

Farrer C, Frey SH, Van Horn JD, Tunik E, Turk D, Inati S, Grafton ST (2008) The angular gyrus computes action awareness representations. Cereb Cortex 18:254-261.

Fierro B, Palermo A, Puma A, Francolini M, Panetta ML, Daniele O, Brighina F (2007) Role of the cerebellum in time perception: a TMS study in normal subjects. J Neurol Sci 263:107-112.

Friston KJ, Penny WD, Glaser DE (2005) Conjunction revisited. Neuroimage 25:661-667.

Frith CD, Blakemore SJ, Wolpert DM (2000) Abnormalities in the awareness and control of action. Philos Trans R Soc Lond B Biol Sci 355:1771-1788.

Genovese CR, Lazar NA, Nichols T (2002) Thresholding of statistical maps in functional neuroimaging using the false discovery rate. Neuroimage 15:870-878.
Geyer S, Ledberg A, Schleicher A, Kinomura S, Schormann T, Bürgel U, Klingberg T, Larsson J, Zilles K, Roland PE (1996) Two different areas within the primary motor cortex of man. Nature 382:805-807.

Geyer S, Schleicher A, Zilles K (1999) Areas 3a, 3b, and 1 of human primary somatosensory cortex. Neuroimage 10:63-83.

Geyer S, Schormann T, Mohlberg H, Zilles K (2000) Areas 3a, 3b, and 1 of human primary somatosensory cortex. Part 2 . Spatial normalization to standard anatomical space. Neuroimage 11:684-696.

Grèzes J, Armony JL, Rowe J, Passingham RE (2003) Activations related to "mirror" and "canonical" neurones in the human brain: an fMRI study. Neuroimage 18:928-937.

Guzzetta A, Staudt M, Petacchi E, Ehlers J, Erb M, Wilke M, Krägeloh-Mann I, Cioni G (2007) Brain representation of active and passive hand movements in children. Pediatr Res 61:485-490.

Ivry RB, Spencer RM, Zelaznik HN, Diedrichsen J (2002) The cerebellum and event timing. Ann N Y Acad Sci 978:302-317.

Jeannerod M (2008) The sense of agency and its disturbances in schizophrenia: a reappraisal. Exp Brain Res. Advance online publication. Retrieved November 5, 2008. doi: 10.1007/s00221-008-1533-3

Kawato M (1999) Internal models for motor control and trajectory planning. Curr Opin Neurobiol 9:718-727.

Keysers C, Perrett DI (2004) Demystifying social cognition: a Hebbian perspective. Trends Cogn Sci 8:501-507.

Keysers C, Wicker B, Gazzola V, Anton JL, Fogassi L, Gallese V (2004) A touching sight: SII/PV activation during the observation and experience of touch. Neuron 42:335-346.

Koski L, Iacoboni M, Dubeau MC, Woods RP, Mazziotta JC (2003) Modulation of cortical activity during different imitative behaviors. J Neurophysiol 89:460-471.

Lee KH, Egleston PN, Brown WH, Gregory AN, Barker AT, Woodruff PW (2007) The role of the cerebellum in subsecond time perception: evidence from repetitive transcranial magnetic stimulation. J Cogn Neurosci 19:147-157.

MacDonald PA, Paus T (2003) The role of parietal cortex in awareness of self-generated movements: a transcranial magnetic stimulation study. Cereb Cortex 13:962-967.

Menon V, Glover GH, Pfefferbaum A (1998) Differential activation of dorsal basal ganglia during externally and self paced sequences of arm movements. Neuroreport 9:1567-1573.

Nichols T, Brett M, Andersson J, Wager T, Poline JB (2005) Valid conjunction inference with the minimum statistic. Neuroimage 25:653-660.

Robinson CJ, Burton H (1980) Somatic submodality distribution within the second somatosensory (SII), 7b, retroinsular, postauditory, and granular insular cortical areas of M. fascicularis. J Comp Neurol 192:93-108.

Roy JE, Cullen KE (2004) Dissociating self-generated from passively applied head motion: neural mechanisms in the vestibular nuclei. J Neurosci 24:2102-2111.

Schütz-Bosbach S, Mancini B, Aglioti SM, Haggard P (2006) Self and other in the human motor system. Curr Biol 16:1830-1834.

Seidler RD, Noll DC, Thiers G (2004) Feedforward and feedback processes in motor control. Neuroimage 22:1775-1783.

Uddin LQ, Iacoboni M, Lange C, Keenan JP (2007) The self and social cognition: the role of cortical midline structures and mirror neurons. Trends Cogn Sci 11:153-157.

von Holst E (1954) Relations between the central nervous system and the perpheral organs. Br J Anim Behav 2:89-94.

Weiller C, Jüptner M, Fellows S, Rijntjes M, Leonhardt G, Kiebel S, Müller S, Diener HC, Thilmann AF (1996) Brain representation of active and passive movements. Neuroimage 4:105-110.

Whitsel BL, Petrucelli LM, Werner G (1969) Symmetry and connectivity in the map of the body surface in somatosensory area II of primates. J Neurophysiol 32:170-193.

Wolpert DM, Flanagan JR (2001) Motor prediction. Curr Biol 11:R729-R732.

Wolpert DM, Ghahramani Z, Jordan MI (1995) An internal model for sensorimotor integration. Science 269:1880-1882. 